\title{
Proposta de instrumento para avaliar conhecimento de jovens sobre métodos contraceptivos
}

\author{
Nancy Capretz Batista da Silva \\ Thiago Bomfim \\ Nilceu Pfitter Cardozo \\ Maria Aparecida Paiva Franco \\ Susi Lippi Marques \\ Universidade Federal de São Carlos, São Carlos-SP, Brasil
}

\begin{abstract}
Resumo: No Brasil, as ocorrências de gravidez na adolescência têm gerado discussões e preocupação no âmbito da saúde pública. Visto que a associação com o uso inadequado de métodos contraceptivos é direta, torna-se essencial avaliar o conhecimento de adolescentes sobre tais métodos para intervir adequadamente. Este estudo realizou este levantamento e avaliou a eficácia de um questionário construído para medir tal conhecimento de estudantes do nível médio de uma cidade do interior de São Paulo. Foi encontrada falta de conhecimento adequado sobre os métodos contraceptivos (como são, uso, eficácia, indicação), independente do sexo e do tipo de escola. O instrumento apresentou níveis psicométricos satisfatórios, adequado para ser disponibilizado e os dados obtidos por meio dele mostram-se confiáveis. Sua utilização enquanto recurso técnico de avaliação poderá servir como apoio para a elaboração de intervenções que almejem maior conhecimento sobre métodos contraceptivos, sexo seguro e menores taxas de gravidez na adolescência.
\end{abstract}

Palavras-chave: Questionário. Gravidez na adolescência. Métodos contraceptivos.

\section{Proposal of an instrument to evaluate youths' knowledge on contraceptive methods}

Abstract: In Brazil, the occurrence of pregnancy in adolescence has generated discussions and concern in the public health area. Considering that the association with inadequate use of contraceptive methods is direct, it is essential to evaluate adolescents' knowledge regarding these methods to intervene appropriately. This study aimed to evaluate the efficacy of a questionnaire built to measure the knowledge of high schools students in a city in the interior of Sao Paulo. Lack of adequate knowledge about contraceptive methods (how they are, use, efficacy, indication) was found, despite gender and type of school. The instrument presented satisfactory psychometric levels, reliability and appropriateness to be used as evaluation tool. Its use can support the elaboration of interventions towards improved knowledge on contraceptive methods, safe sex, and reduced rates of pregnancy in adolescence.

Keywords: Questionnaire. Adolescent pregnancy. Contraceptive devices.

\section{Propuesta de un instrumento para evaluar el conocimiento de los jovenes sobre los métodos contraceptivos}

Resumen: En Brasil el embarazo en la adolescencia viene generando esfuerzos de prevención desde la salud pública. Debido a su asociación directa con el uso inadecuado de métodos contraceptivos se torna esencial, para lograr una intervención adecuada, el indagar sobre el conocimiento que los adolescentes tienen sobre dichos métodos. Así, además de presentar esta información, este trabajo analiza la eficacia de un cuestionario construido para evaluar tal conocimiento a partir de una muestra de 406 estudiantes del nivel medio, pertenecientes a escuelas públicas y privadas de una ciudad del interior paulista. Se realizó un análisis comparativo de los grupos considerando los puntajes obtenidos en la encuesta, así como la temática de los ítems empleados. Se encontró una falta de conocimientos adecuados sobre los métodos contraceptivos (cómo son, uso, eficacia, recomendación), independientemente del sexo y tipo de escuela considerada. El instrumento presentó niveles psicométricos satisfactorios y podrá servir como apoyo para la elaboración de programas de intervención que brinden un mayor conocimiento sobre los métodos contraceptivos, sexo seguro y menores tasas de embarazo en la adolescencia.

Palabras clave: Cuestionario. Embarazo en la adolescencia. Métodos contraceptivos. 


\section{Introdução}

A gravidez na adolescência é definida pela Organização Mundial da Saúde como aquela que ocorre entre os dez e os vinte anos incompletos. Ela tem sido qualificada como "precoce" e "indesejada" pelo desvio ou transtorno que representa para a vida do(a) jovem (Mena, 2005; Costa e cols., 2005). "Riscos biopsicossociais" tanto para a mãe quanto para seus filhos são salientados na gravidez precoce, constituindo como "problema social" ou de saúde pública, gerando, assim, esforços das políticas públicas para ou "prevenir" ou "coibir" este tipo de gravidez (Cabral, 2003).

A gravidez na adolescência não é um fenômeno recente (Aquino e cols., 2003), contudo, apesar da queda observada nas taxas de fecundidade, a análise desta por faixas etárias específicas indica um aumento significativo no intervalo que tange dos 15 aos 19 anos de idade (Cabral, 2003; Goldenberg, Figueiredo \& Souza e Silva, 2005). De acordo com o Instituto Brasileiro de Geografia Estatística (IBGE) (2002), a "média de idade da fecundidade da mulher brasileira diminuiu acentuadamente de 1980 para 2000", e "dados do Censo 2000 indicam uma elevação da contribuição da fecundidade das mulheres mais jovens na fecundidade total, isto é, considerado o total de filhos de todas as mulheres em idade fértil, aumentou o percentual de filhos das jovens entre 15 e 19 anos nesse total".

A Fundação Sistema Educacional de Análise de Dados (SEADE) (2003) aponta que no país a fecundidade das adolescentes é elevada se comparada com a européia ou a japonesa e que entre 1980 e 2002, houve uma sensível redução da fecundidade por faixas etárias, exceto no grupo de 15 a 19 anos, que manteve-se praticamente no mesmo nível no estado de São Paulo, ao redor de 70 filhos anuais para cada mil jovens. No início deste século, a estimativa apresenta uma pequena queda.

Estariam associados à gravidez na adolescência: a tendência de queda da idade média da menarca e da iniciação sexual, a falta de informação sobre métodos contraceptivos e a dificuldade de acesso a estes (Cabral, 2003). Outras variáveis que poderiam ser associadas à fecundidade no período seriam nível de escolaridade e socioeconômico baixo, cor, estado civil e o desconhecimento da fisiologia reprodutiva, como a capacidade de identificação do período fértil (Belo \& Pinto e Silva, 2004). Foi encontrado que a ocorrência de uma gravidez antes dos vinte anos variou inversamente com a renda e a escolaridade e, constatou-se uma maior prevalência de gravidez na adolescência entre homens e mulheres negros, pardos e indígenas em relação aos declarados brancos (Aquino e cols., 2003). Dentre múltiplas determinações, a erotização do adolescente, promovida pela mídia, estimula a iniciação sexual precoce que, na ausência do domínio das práticas contraceptivas, pode resultar em gravidez não desejada (Goldenberg e cols., 2005). Em países como Brasil, México e Chile, há reconhecimento limitado dos direitos sexuais e reprodutivos e as atitudes referentes à sexualidade são conservadoras, particularmente em relação aos adolescentes, o que também pode estar influenciando nos índices de gravidez na adolescência (Díaz, Hardy, Alvarado \& Ezcurra, 2003).

Questões sobre escolaridade e pobreza são colocadas ora no âmbito das conseqüências, ora no das causas do fenômeno (Cabral, 2003; Pirotta \& Schor, 2004; Martins e cols., 2006).

De acordo com a literatura, existe um quadro de "desvantagem social" decorrente da maternidade na adolescência caracterizado pela interrupção prematura da escolaridade, a diminuição da capacidade de competir no mercado de trabalho e a maior instabilidade nas relações conjugais (Cabral, 2003). Além disso, existe a perda de aproveitamento pleno das oportunidades para completo desenvolvimento pessoal e até mesmo para ter opções de vida e oportunidades para um pleno desenvolvimento pessoal a que se tem direito (Belo \& Pinto e Silva, 2004). Ainda podemos citar a maior probabilidade dessas adolescentes acabarem como dependentes do assistencialismo governamental e o fato de que muitas delas ainda precisam de suas mães; estão despreparadas para assumir as responsabilidades psicológicas, sociais e econômicas e; apresentam conhecimento pouco realista sobre as necessidades e capacidades de um bebê (Newcombe, 1999). Ao mesmo tempo, há indícios, no plano biológico-social, de maior concentração de agravos à saúde materna 
e complicações perinatais (baixo ganho de peso materno, a desproporção céfalo-pélvica, a préeclampsia, a prematuridade, o baixo peso ao nascer e o Apgar baixo no quinto minuto), particularmente entre adolescentes mais jovens (Goldenberg e cols., 2005).

Além de todos esses fatores, aspectos como baixa auto-estima, ausência de apoio familiar, alto nível de estresse, poucas expectativas frente ao futuro e presença de sintomas depressivos, além de comuns, exercem influência na maneira como a relação entre a gestante adolescente e seu bebê irá se constituir (Sabroza, Leal, Souza Jr \& Nogueira da Gama, 2004).

Autores brasileiros têm mostrado que a gravidez indesejada chega a uma proporção de 50\% entre adolescentes de 15 a 19 anos, como verificado no Centro de Atenção Integral à Saúde da Mulher (CAISM Universidade Estadual de Campinas), que foi de $45,9 \%$. O principal motivo relatado por essas jovens para sua ocorrência foi o não uso de métodos anticoncepcionais. Um estudo levantou entre 4.634 jovens de três capitais brasileiras, de três regiões distintas, relato de $55,1 \%$ dos homens e $27,9 \%$ das mulheres de gravidez entre adolescentes (Aquino e cols., 2003). Entre estes, a maioria ( $85,6 \%$ dos homens e 70,3\% das mulheres) não pretendia engravidar, nem pensava no assunto; porém, apenas $36,6 \%$ dos rapazes e $31,4 \%$ das moças relataram uso de contracepção nessa ocasião.

Os obstáculos existentes para o uso consistente dos métodos contraceptivos, especialmente para as adolescentes mais jovens, incluem as pressões sociais e os papéis de gênero (Cabral, 2003). Entre eles, destacam-se: a objeção de seu uso pelo parceiro, "o pensar que não engravidaria" (pensamento característico do período adolescente), ou por "não esperar ter relações naquele momento" (Belo \& Pinto e Silva, 2004).

O comportamento contraceptivo é sempre posterior ao início do relacionamento sexual com a parceira. Alega-se que é atribuição exclusiva da mulher a responsabilidade com relação à vida reprodutiva e atribui-se à imprevisibilidade das relações o não-uso da contracepção. Mais uma vez, atributos de gênero desempenham seu papel nesse cenário. "A própria literatura na saúde coletiva tem assinalado que a sexualidade masculina, pelo menos na cultura sexual brasileira, é freqüentemente representada como 'incontrolável' e, portanto, ser homem significa ter menos controle sobre seus impulsos sexuais" (Cabral, 2003, p. 288). Verificouse que a falta de responsabilidade masculina no comportamento sexual adolescente se deve ao foco que os estudos concentram no papel da mulher sobre a fertilidade e em como os programas comunitários e políticas públicas influenciam a atividade sexual e as decisões da mulher sobre fertilidade, deixando de lado o papel masculino no comportamento sexual adolescente (Huang \& Han, 2004).

Além disso, a maior parte dos estudos sobre gravidez na adolescência "aborda as questões relacionadas ao sexo feminino, possivelmente, resultado da influência sociocultural, na qual a mulher é considerada a principal responsável pela gestação e cuidado com a criança" (Costa e cols., 2005, p. 720).

Assim, apesar de todos os benefícios gerados pelo uso dos métodos contraceptivos para as brasileiras, esses não foram conquistados para sua autonomia, mas a gerou de certa forma. Este fato pode ter relação com o modo como jovens e profissionais lidam com o tema, pois nem sempre a autonomia e o direito de escolha quanto à reprodução são buscados e ensinados de forma devida (Pedro, 2003).

Sumarizando, os métodos contraceptivos não têm atingido os adolescentes da forma como deveriam, mas existem em grande número. Eles visam evitar a gravidez, agindo de várias formas e, alguns impedem a transmissão de doenças. A escolha de um método contraceptivo deve ser baseada na consulta ao médico, pois ele indica aquele que melhor se adapta às necessidades individuais, sem causar problemas ao organismo (Comunidade Médica, 2004).

Como grande preocupação para a saúde pública do país, os índices ainda elevados de gestação adolescente vem acompanhado do fato de a adolescência ser uma faixa etária de alto risco para doenças sexualmente transmissíveis. Martins e cols. (2006) chegam até mesmo a dizer que entre os adolescentes está a maior incidência dessas doenças, porém, o IBGE aponta que no Brasil esses dados ainda são bastante imprecisos. Com relação à infecção pelo HIV, estima-se em 597.443 o número 
de infectados no Brasil, na faixa etária de 15 a 49 anos, no ano 2000 (Brasil, 2004). Decorre daí a importância de focalizarmos o conhecimento e uso de métodos contraceptivos nessa população, não apenas pela influência que isso tem na gestação adolescente como também na prevenção de doenças.

Com o crescente reconhecimento da gravidez na adolescência como um problema de saúde pública, é possível direcionar a intervenção dos profissionais de saúde que trabalham com essa população através do conhecimento de métodos eficazes de prevenção e das condições culturais e sócio-econômicas, para a busca de melhores índices (positivos) na saúde pública.

Desse modo, este trabalho teve como objetivo a elaboração de um instrumento válido e fidedigno para avaliar o conhecimento de adolescentes sobre métodos contraceptivos por meio de um teste objetivo, para que este possa levantar informações para consecução de uma análise mais objetiva das condições nas quais se encontram os membros desta população, assim como o seu conhecimento sobre o tema. No âmbito prático este instrumento de avaliação poderia vir a ser utilizado como recurso técnico e auxiliar no planejamento de programas de intervenção e prevenção de gravidez na adolescência no Brasil.

\section{Método}

\section{Participantes}

Na avaliação dos parâmetros psicométricos do instrumento a ser testado participaram 406 estudantes do ensino fundamental e médio de escolas públicas e particulares da cidade de São Carlos-SP. Entre eles, 40 eram rapazes de escolas públicas (REPUs), 113 eram garotas de escolas públicas (GEPUs), 87 eram rapazes de escolas particulares (REPAs), e 166 eram garotas de escolas particulares (GEPAs).

\section{Material}

Foi utilizado como instrumento um teste objetivo composto por 18 itens de múltipla escolha sobre conhecimentos a respeito de métodos contraceptivos. Este instrumento foi construído e testado em uma amostra piloto em estudo anterior (Viana \& Marques, 2001; Bomfim, Cardozo \& Marques, 2002), tendo passado por uma análise teórica, Análise Semântica feita com uma amostragem similar ao do estudo $(n=30$ participantes) e Análise de Conteúdo realizada por profissionais da área médica especializados ginecologistas e obstetras. Após a aplicação em amostra piloto ( $\mathrm{n}=30$ participantes) as respostas foram analisadas e foram obtidos índices psicométricos satisfatórios. O índice de dificuldade dos itens variou entre 0,30 e 0,70, a Discriminabilidade variou entre $60 \%$ e $80 \%$ e a Precisão, obtida através da técnica Alfa de Cronbach, corrigida pela fórmula de Spearman-Brown, foi de 0,69 (Pasquali, 1997; Cronbach, 1996). Desta forma, o referido instrumento mostrou-se adequado para aplicação tanto em relação ao conteúdo de seus itens quanto à clareza de entendimento dos mesmos. A partir deste estudo inicial, imprescindível no processo de proposição de instrumentos de medida, este instrumento apontou para a viabilidade de sua aplicação em amostra expandida, proposta deste estudo.

Uma folha de rosto com instruções e esclarecimentos sobre o preenchimento do teste acompanhava o instrumento. Além disso, para caracterização da amostra, havia um questionário de dados pessoais e informações sobre utilização de contraceptivos, veículos e fontes de informações e o quanto os participantes consideravam-se informados sobre o tema.

\section{Procedimento}

Após a obtenção da autorização da direção das diferentes escolas, os estudantes foram contatados e, mediante a exposição dos objetivos do estudo, aderiam ou não à participação na pesquisa. A aplicação do instrumento e do questionário, na amostra de 406 estudantes, foi realizada nas salas de aula e pátio das escolas. Em alguns casos os participantes preferiram responder em outros locais e horários, sendo então agendados dia, horário e local conveniente.

Os dados do questionário preliminar foram descritos por análise qualitativa e quantitativa. Foi realizada uma análise comparativa dos grupos amostrais para os escores obtidos no teste e, por último, uma análise dos itens do teste em função de sua temática de acordo com os acertos gerais obtidos e tendências grupais. 


\section{Considerações éticas}

Este estudo contou com a participação voluntária de estudantes, que após autorização da direção das escolas, foram esclarecidos quanto ao objetivo da pesquisa, isto é, sua colaboração no processo de avaliação do instrumento, foco deste estudo. Os participantes foram informados sobre a possibilidade de interromper a aplicação caso desejassem, sendo que, neste caso, seus dados não seriam considerados na pesquisa. Foi assegurado aos colaboradores o anonimato e a sua identidade salvaguardada no caso da publicação dos resultados. Todos os cuidados éticos foram adotados visando à integridade e bem-estar dos participantes, conforme estabelecido pela resolução 196/96 do Ministério da Saúde.

\section{Resultados}

\section{Análise de parâmetros psicométricos oriundos da amostra com 406 participantes}

Os resultados obtidos, a partir dos 406 instrumentos respondidos, apontaram uma boa variação nos Índices de Dificuldade (ID) dos itens entre $0,18-0,78$ e mais de $60 \%$ dos itens tiveram uma discriminação mediana (entre $35 \%$ e $65 \%$ ), sendo que apenas um item apresentou discriminação inferior a $20 \%$ (18,52\% de discriminação). Para o cálculo da discriminação dos itens, utilizou-se a Estatística D com grupos-critério superior e inferior (regra 27 de Kelley). O coeficiente alfa de Cronbach encontrado foi de 0,62, o que remete a um bom índice de fidedignidade (precisão) do teste. De acordo com os parâmetros psicométricos obtidos o instrumento mostrou-se adequado para aplicação (Pasquali, 1997; Cronbach, 1996).

\section{Dados do questionário: Panorama da amostra}

Os adolescentes que responderam o questionário estavam na faixa etária de 13 a 24 anos, havendo uma concentração entre 14 e 16 anos, 77\% (GEPUs), 60\% (REPUs), 84\% (GEPAs) e 75\% (REPAs). Apesar de a adolescência, segundo Organização Mundial de Saúde, abranger a faixa etária entre os dez e os 20 anos incompletos, foram incluídos os dados de quatro jovens que faziam parte das turmas que participaram do estudo com idade entre 20 e 24 anos.

O levantamento realizado por meio das questões abertas evidenciou que 50\% dos estudantes acreditam estar bem informados sobre o assunto 44\% (GEPUs), 45\% (REPUs), 52\% (GEPAs) e 56\% (REPAs), sendo que apenas $15 \%$ não se considerou bem informado e $29 \%$ considerou que ainda desconheciam algumas informações - 6\% dos adolescentes não responderam essa questão.

Entre eles, 82\% [89\% (GEPUs), 88\% (REPUs), 79\% (GEPAs) e 76\% (REPAs)] declarou ter recebido orientação acerca de métodos contraceptivos, sendo que a escola é o local mais indicado como fonte dessa orientação [79\% das respostas: $85 \%$ (GEPUs), 85\% (REPUs), 78\% (GEPAs) e 70\% (REPAs)]. Outras fontes de orientação estariam em casa $(7 \%)$, em cursos e palestras (3\%), na mídia ( $2 \%)$ e na consultas médicas $(1 \%)$.

Apenas 20\% dos adolescentes declararam o uso de métodos contraceptivos [19\% (GEPUs), 55\% (REPUs), 12\% (GEPAs) e 21\% (REPAs)], valendo destacar que $78 \%$ deles não consideram possuir vida sexual ativa. E ainda, seis adolescentes declararam não fazer uso de métodos contraceptivos e um relatou que o uso depende da situação. Apesar de a maioria dos estudantes não ter declarado vida sexual ativa, quando perguntado qual o método utilizado, $14 \%$ deles indicaram o uso de preservativo masculino e $3 \%$ a associação do preservativo masculino com o contraceptivo oral.

Entre os métodos contraceptivos mais conhecidos por estes adolescentes estão o preservativo masculino [ $88 \%$, sendo $85 \%$ (GEPUs), $93 \%$ (REPUs), 87\% (GEPAs) e 93\% (REPAs)] e o contraceptivo oral [87\%, sendo 86\% (GEPUs), $80 \%$ (REPUs), 91\% (GEPAs) e 83\% (REPAs)]. Outros métodos citados foram: DIU (51\%), diafragma (40\%), camisinha feminina (34\%), tabelinha (22\%), coito interrompido (9\%), vasectomia (9\%), laqueadura de trompas $(9 \%)$, pílula do dia seguinte $(6 \%)$, espermicida (3\%) e anticoncepcional injetável (1\%).

Posto isto, pode-se apreender que os dados oriundos do questionário inicial permitem traçar um 
panorama e caracterizar os participantes necessitando informações adicionais a serem consideradas no planejamento preventivo e interventivo.

\section{Análise comparativa dos grupos amostrais}

Uma análise estatística realizada a partir da aplicação do Teste $t$ para comparações de médias dos escores obtidos no teste objetivo revelou que há uma pequena evidência de diferença entre algumas das três primeiras comparações realizadas entre: Alunos - Esc.Pública X Esc.Particular [ $\mathrm{t}=-2,283$; 375gl; $(p=0,023)]$; Gênero - Homem Esc.Pública X Mulher Esc.Pública $[\mathrm{t}=-2,387 ; 77 \mathrm{gl}(p=0,019)] \mathrm{e}$ Homem X Mulher $[\mathrm{t}=-2,232 ; 257 \mathrm{gl}(p=0,026)]$. Não foi encontrada diferença para a comparação Gênero - Homem Esc.Particular X Mulher Esc.Particular $[\mathrm{t}=-2,232 ; 175 \mathrm{gl}(p=0,30)]$. Contudo, em função das múltiplas comparações entre os escores obtidos aplicou-se a correção de Bonferroni para checar de maneira rigorosa a existência de diferença significativa entre as comparações efetuadas que neste caso, deveria ser $p=0,12$ valor este não encontrado neste trabalho. Desta forma, pode-se concluir que o nível de conhecimento desta amostra sobre métodos contraceptivos parece ser igual e independe do tipo de escola e sexo. Na Tabela 1 encontram-se os escores gerais do teste para a amostra dividida em seus grupos de participantes.

Tabela 1

Amplitude dos escores e escore médio para os grupos de participantes e geral

\begin{tabular}{lllcrr}
\hline & Grupos & \multicolumn{2}{c}{ Amplitude Dos Escores } & \multicolumn{2}{c}{ Escore Médio } \\
\hline Escolas & Rapazes & $2-17$ & $2-12$ & 8,84 & 7,88 \\
Públicas & Garotas & & $3-17$ & & 9,19 \\
Escolas & Rapazes & $2-18$ & $2-15$ & 8,86 & 8,61 \\
Particulares & Garotas & & $3-18$ & & 8,99 \\
\hline GERAL & & $2-18$ & & 8,85 \\
\hline
\end{tabular}

\section{Análise dos itens do teste objetivo em função da sua temática}

O número de acertos em cada item e a posterior análise do conteúdo dos itens de acordo com o grau de dificuldade que apresentaram para o grupo de participantes foi investigado. Os acertos dos participantes por itens, em porcentagem, podem ser observados na Tabela 2 .

Entre os dezoito itens que compunham o instrumento, o item 10 (forma, eficácia e uso da camisinha feminina) foi o de maior porcentagem de acerto, no geral (81\%). Os itens 1 (o que são os métodos contraceptivos), 3 (formas corretas sobre o uso da camisinha masculina), 4 (uso, indicação e prevenção do DIU) e 17 (uso da pílula anticoncepcional, da esponja vaginal, da tabelinha e do DIU) apresentaram uma boa porcentagem de acertos (66\%, 72\%, 73\% e 65\%, respectivamente). Os itens 2 (uso de DIU, pílula anticoncepcional convencional, camisinha feminina, camisinha masculina e dia- fragma), 6 (uso eficaz da pílula anticoncepcional), 7 (eficácia e uso do diafragma), 9 (em que consiste o coito interrompido, sua eficácia e conseqüências para o ato sexual), 11 (uso da pílula do dia seguinte), 15 (eficácia de pílula, DIU, camisinha e anticoncepcional injetável) e 16 (ocorrência de gravidez em detrimento de algumas condições - virgindade, uso de ducha, entre outras - e o uso da camisinha feminina) tiveram uma porcentagem de acertos que indica domínio de aproximadamente metade dos participantes $(57 \%$, $59 \%, 43 \%, 56 \%, 44 \%, 56 \%$ e $40 \%$, respectivamente). Os itens 5 (tempo decorrente para a eficácia da pílula anticoncepcional), 12 (uso e a atuação do anticoncepcional vaginal), 13 (conseqüências dos métodos cirúrgicos - vasectomia e laqueadura de trompas), 14 (amamentação como método contraceptivo) e 18 (índice de segurança dos métodos contraceptivos) parecem ter sido difíceis, visto que suas porcentagens de acerto foram 31, 23, 30, 35 e 29 , respectivamente. O item 8 (uso da tabelinha) apresentou a menor porcentagem de acerto, $18 \%$. 
Tabela 2

Acertos dos participantes por itens em porcentagem por grupo e geral

\begin{tabular}{cccccc}
\hline Itens GEPUs REPUs GEPAs REPAs & $\begin{array}{c}\text { Geral } \\
(\mathrm{n}=113)\end{array}$ \\
\hline$l$ & 63 & 60 & 69 & 67 & 66 \\
2 & 58 & 50 & 60 & 52 & 57 \\
3 & 73 & 83 & 67 & 75 & 72 \\
4 & 79 & 68 & 70 & 71 & 73 \\
5 & 27 & 30 & 40 & 23 & 31 \\
6 & 66 & 65 & 57 & 51 & 59 \\
7 & 39 & 48 & 43 & 45 & 43 \\
8 & 16 & 10 & 19 & 21 & 18 \\
9 & 58 & 48 & 58 & 56 & 56 \\
10 & 88 & 73 & 78 & 83 & 81 \\
$l 1$ & 54 & 30 & 43 & 39 & 44 \\
12 & 19 & 18 & 24 & 30 & 23 \\
13 & 39 & 20 & 27 & 29 & 30 \\
14 & 35 & 28 & 35 & 38 & 35 \\
15 & 54 & 40 & 61 & 56 & 56 \\
16 & 43 & 43 & 37 & 38 & 40 \\
17 & 72 & 63 & 61 & 67 & 65 \\
18 & 36 & 15 & 33 & 22 & 29 \\
\hline & & & & &
\end{tabular}

Ao ser considerada esta análise temática tornase possível a apreensão de quais informações necessitariam ser transmitidas/esclarecidas, além de se verificar o nível de conhecimento que a amostra e/ou população que está sendo foco de um programa ou orientação possui sobre o tema.

\section{Discussão}

A partir dos resultados encontrados podemos fazer importantes considerações e contribuir para a intervenção com adolescentes para prevenir a gravidez nesta fase do desenvolvimento, assim como esclarecer o uso e a eficácia dos métodos contraceptivos para esta população baseando-se em dados de um instrumento (teste objetivo) que se mostrou adequado para este propósito. Analisando os resultados de maneira geral, pode-se concluir que os índices psicométricos encontrados foram satisfatórios e mantiveram-se estáveis quando aplicados em amostra expandida $(\mathrm{n}=406)$. Deste modo, pode-se perceber que o instrumento construído e proposto por este estudo mostra-se adequado para propiciar subsídios para planejamento e avaliações de intervenções que possam ser realizadas tanto no âmbito da saúde pública quanto de outras áreas, por exemplo, educacional.

Baseados no desempenho da amostra testada $(n=406)$ para a proposição deste teste objetivo, podemos verificar que os adolescentes participantes não apresentam conhecimento adequado sobre os métodos contraceptivos - como são, seu uso, sua eficácia, sua indicação, apontando necessidade de intervenção adequada com esta população e mudança de atitude de pais e profissionais que discutem o assunto, já que estas são as fontes de orientação relatadas pela amostra participante deste estudo. Esse dado vai de encontro com o relato de Martins e cols. (2006) de que a compreensão da prevenção das doenças sexualmente transmissíveis entre os adolescentes é escassa e insuficiente para promover um comportamento sexual seguro. Sobre a sexualidade na adolescência, Parera e Surís (2004) sugerem que o bom relacionamento com a mãe não tem impacto sobre o comportamento contraceptivo na adolescente. Apesar de os pais poderem influenciar o comportamento sexual dos filhos, muitos encontram dificuldades em iniciar essas discussões e os filhos freqüentemente sentem-se desconfortáveis com perguntas pessoais (Romo, Nadeem, Au \& Sigman, 2004). Assim, sugerem que os pais se beneficiariam se fossem aconselhados em como fazer perguntas pessoais para influenciar o comportamento sexual dos filhos adolescentes. Neste caso, programas preventivos e de orientação deveriam contemplar também orientações voltadas para pais.

Os dados obtidos no questionário apontam que uma parcela razoável desta população é consciente de sua falta de informação, visto que $15 \%$ deles não se considerou bem informados e $29 \%$ deles considerou que ainda desconheciam algumas informações. Este fato é preocupante, pois a literatura (Cabral, 2003) aponta a relação entre a falta de conhecimento sobre métodos contraceptivos e a incidência de gravidez na adolescência. 
A baixa porcentagem de adolescentes que revelaram o uso de métodos contraceptivos neste estudo está claramente relacionada ao relato da maioria destes adolescentes de não possuir vida sexual ativa. É esperado que o comportamento contraceptivo dessa população seja posterior ao início do relacionamento sexual, assim como sugere Cabral (2003).

Apesar de a mídia não ser a fonte de orientação mais citada, pode-se observar que o método contraceptivo mais conhecido por estes adolescentes, o preservativo masculino, é aquele mais divulgado pelos veículos de informação. Assim, essa amostra apresentou um conhecimento muito limitado ao preservativo masculino e ao anticoncepcional oral. Porém, a maior porcentagem de acertos no instrumento foi encontrada no item 10, que versava sobre a forma, eficácia e uso da camisinha feminina, mostrando que apesar de não ser tão popular quanto a camisinha masculina, já é conhecida pelos participantes do estudo.

A baixa porcentagem de acertos no item 8 , que debatia o uso da tabelinha, evidencia que esse método é pouco conhecido por esta população, portanto, não seria indicado como método contraceptivo para esses adolescentes. Mais importante ainda é a baixa precisão desse método e o fato de não proteger das doenças sexualmente transmissíveis, tornando-o pouco recomendável.

Não foi encontrada diferença significativa entre tipos de instituição de ensino - pública ou particular - e conhecimento sobre métodos contraceptivos, assim como também não foi encontrada diferença significativa entre garotas e rapazes quanto ao conhecimento sobre o assunto estudado. Porém, um estudo (Martins e cols., 2006) mostrou que características como maior nível de escolaridade (ensino médio), sexo feminino, estudar em escola privada, ser branco(a) e ser solteiro(a) foram fatores associados positivamente a um maior conhecimento. Esses dados devem ser levados em consideração na elaboração de intervenções com adolescentes. Talvez características não levantadas no instrumento do presente estudo devam ser consideradas.

Os dados deste estudo oriundos do instrumento proposto sugerem que as orientações fornecidas aos adolescentes parecem não estar atingindo de maneira eficiente a amostra em questão, refletindo a falta de apoio das orientações em dados reais sobre o conhecimento dessa população. Considerando as reflexões que vêm sendo feitas sobre a gravidez na adolescência, e o caráter preventivo que as intervenções devem assumir, ter acesso ao conhecimento de adolescentes sobre os métodos contraceptivos parece ser uma via interessante de planejamento dessas intervenções.

Assim, o instrumento mostrou-se bastante útil para verificar o nível de conhecimento de adolescentes sobre métodos contraceptivos. Neste sentido, o instrumento fornece informações específicas sobre o conhecimento de uma determinada amostra e viabiliza um planejamento interventivo específico para atender às suas necessidades. Além disso, tem-se a possibilidade de sua utilização em fase posterior de implantação do programa de intervenção, com o propósito de avaliar sua abrangência/impacto. Ainda, como recurso técnico de avaliação este instrumento poderia ser utilizado no setor público em programas de atendimento e orientação de adolescentes e em programas de orientação sexual oferecidos pelas escolas.

Portanto, dentro de suas possibilidades informativas, a presente investigação pretendeu oferecer uma contribuição metodológica ao estudo do tema sobre gravidez na adolescência e conhecimento de métodos contraceptivos, compartilhando algumas evidências baseadas no conhecimento de uma amostra de jovens da faixa etária em questão e proposição de um instrumento avaliativo.

\section{Referências}

Aquino, E. M. L., Heilborn. M. L., Knauth, D., Bozon, M., Almeida, M. C., Araújo J., \& Menezes, G. (2003). Adolescência e reprodução no Brasil: A heterogeneidade dos perfis sociais. Cadernos de Saúde Pública, 19(Supl. 2), 377-388.

Belo, M. A. V., \& Pinto e Silva, J.L. (2004). Conhecimento, atitude e prática sobre métodos anticoncepcionais entre adolescentes gestantes. Revista de Saúde Pública, 38, 479-487. 
Bomfim, T., Cardozo, N. P., \& Marques, S. L. (2002). Instrumento objetivo de mensuração psicológica do conhecimento de adolescentes acerca de métodos contraceptivos: Uma análise psicométrica [Resumo]. In Sociedade Brasileira de Psicologia (Org.), Reunião Anual de Psicologia, 32 (p. 411). Florianópolis: SBP.

Brasil. Ministério da Saúde. Programa Nacional de DST e Aids. (2004). Política de distribuição de preservativos para ações de prevenção das DST/HIV/Aids no Brasil. Brasília, DF. Retirado 12 de janeiro de 2007, de http://www.aids.gov.br/ final/prevencao/preservativo.pdf.

Cabral, C. S. (2003). Contracepção e gravidez na adolescência na perspectiva de jovens pais de uma comunidade favelada do Rio de Janeiro. Cadernos de Saúde Pública, 19(Supl. 2), 283-292.

Comunidade Médica (2004). Contraceptivos. ENews. Retirado 17 de novembro 2004, de http:// www.comunidademedica.com.br/ saude_mulher_news.php?lista $=2$.

Costa, M. C. O., Lima, I. C., Júnior, D. F. M., Santos, C. A. S. T., Araújo, F. P. O., \& Assis, D. R. (2005). Gravidez na adolescência e co-responsabilidade paterna: Trajetória sociodemográfica e atitudes com a gestação e a criança. Ciência \& Saúde Coletiva, 10, 719-727.

Cronbach, L. J. (1996). Fundamentos da testagem psicológica. (C. A. Silveira Neto \& M. A. V. Veronese, Trad.) (5a ed.). Porto Alegre: Artes Médicas.

Díaz, S., Hardy, E., Alvarado, G., \& Ezcurra, E. (2003). Acceptability of emergency contraception in Brazil, Chile, and México: 1 - Perceptions of emergency oral contraceptives. Cadernos de Saúde Pública, 19, 1507-1517.

Fundação Sistema Educacional de Análise de Dados. (2003). Estatísticas vitais do Estado de São Paulo: Resenha mensal. Retirado 25 de janeiro 2007, de http://www.seade.gov.br.
Goldenberg, P., Figueiredo, M. C. T., \& Souza e Silva, R. (2005). Gravidez na adolescência, pré-natal e resultados perinatais em Montes Claros, Minas Gerais, Brasil. Cadernos de Saúde Pública, 21, 1077-1086.

Huang, C. C., \& Han, W. J. (2004). Perceptions of child support and sexual activity of adolescent males. Journal of Adolescence, 27, 731-748.

Instituto Brasileiro de Geografia Estatística. (2002). Dados populacionais. Retirado 21 de janeiro 2007, de http://www.ibge.gov.br.

Martins, L. B. M., Costa-Paiva, L. H. S., Osis, M. J. D., Souza, M. H., Pinto-Neto, A. M., \& Tadini, V. (2006). Fatores associados ao uso de preservativo masculino e ao conhecimento sobre DST/AIDS em adolescentes de escolas públicas e privadas do Município de São Paulo, Brasil. Cadernos de Saúde Pública, 22, 315-323.

Mena, F. (2005, 5 de janeiro). Meninas lideram abandono de escola. Folha de São Paulo, Caderno Folha Ribeirão, p. C4.

Newcombe, N. (1999). Desenvolvimento Infantil. In N. Newcombe (Org.), Abordagem de Mussen (pp. 420-422). Porto Alegre: Artes Médicas.

Parera, N., \& Surís, J. C. (2004). Having a good relationship with their mother: A protective factor against sexual risk behavior among adolescent females? Journal of Pediatric and Adolescent Gynecology, 17, 267-271.

Pasquali, L. (1997). Psicometria: Teoria e aplicações. Brasília, DF: Editora Unb.

Pedro, J. M. (2003). A experiência com contraceptivos no Brasil: Uma questão de geração. Revista Brasileira de História, 23(45), 239-260.

Pirotta, K. C. M., \& Schor, N. (2004). Intenções reprodutivas e práticas de regulação da fecundidade entre universitários. Revista de Saúde Pública, 38, 495-502.

Romo, L.F., Nadeem, E., Au, T. K., \& Sigman, M. (2004). Mexican-American adolescents' responsiveness to their mothers' questions about dating and sexuality. Applied Developmental Psychology, 25, 501-522. 
Sabroza, A. R., Leal, M. C., Souza, Jr. P. R., \& Nogueira da Gama, S. G. (2004). Algumas repercussões emocionais negativas da gravidez precoce em adolescentes do município do Rio de Janeiro (1999-2001). Cadernos de Saúde Pública, 20 (Supl. 1), 130-137.

Viana, A. B., \& Marques, S. L. (2001). Construção de instrumentos de avaliação em psicologia. Relatório de Bolsa Treinamento em Construção de instrumentos de avaliação em Psicologia. Manuscrito não publicado. São Carlos: PróReitoria de Graduação, UFSCar.

Artigo recebido em 17/01/2007.

Aceito para publicação em 25/08/2007.

Endereço para correspondência:

Susi Lippi Marques Oliveira. Universidade Federal de São Carlos, CECH - Departamento de Psicologia. Rodovia Washington Luís (SP-310), Km 235. CEP:13565-905, São Carlos-SP, Brasil. E-mail: susilmo@power.ufscar.br

Nancy Capretz Batista da Silva é graduada em Psicologia pela Universidade Federal de São Carlos.

Thiago Bomfim é graduado em Psicologia pela Universidade Federal de São Carlos.

Nilceu Pfitter Cardozo é graduado em Psicologia pela Universidade Federal de São Carlos.

Maria Aparecida Paiva Franco é Professora Associada do Departamento de Estatística da Universidade Federal de São Carlos.

Susi Lippi Marques é Professora Associada do Departamento de Psicologia e docente do Programa de Pós-graduação em Educação Especial da Universidade Federal de São Carlos. 